EPJ Web of Conferences 61, 01003 (2013)

DOI: $10.1051 /$ epjconf/ 20136101003

(C) Owned by the authors, published by EDP Sciences, 2013

\title{
The first two years in the lifetime of the newly born jet associated to Sw J1644+57
}

\author{
A. J. Castro-Tirado ${ }^{1}$, J. L. Gómez ${ }^{1}$, I. Agudo ${ }^{1}$, M. A. Guerrero ${ }^{1}$, M. Bremer ${ }^{2}$, J. M. Winters ${ }^{2}$, J. Gorosabel ${ }^{1}$, \\ R. Sánchez-Ramírez ${ }^{1}$, S. Guziy ${ }^{1}$, M. Jelinek ${ }^{1}$, J. C. Tello ${ }^{1}$, D. Pérez-Ramírez ${ }^{3}$, J. Reyes-Iturbide ${ }^{4}$, I. H. Park ${ }^{5}$, \\ S. Jeong ${ }^{1}$, U. Bach ${ }^{6}$, A. Kraussh ${ }^{6}$, T. P. Krichbaumh ${ }^{6}$ and A. S. Pozanenko ${ }^{7}$ \\ ${ }^{1}$ Instituto de Astrofísica de Andalucía (IAA-CSIC), P.O. Box 03004, E-18080 Granada, Spain. \\ ${ }^{2}$ Institute de Radioastronomie Milimetrique (IRAM), 300 rue de la Piscine, 38406 Saint Martin d'Héres, France. \\ ${ }^{3}$ Facultad de Ciencias Experimentales, Universidad de Jaén, Campus Las Lagunillas, E-23071 Jaén, Spain. \\ ${ }^{4}$ Instituto de Astronomía, Univ. Nacional Autónoma de México, Apdo. Postal 70-264, 04510 México, D. F., México. \\ 5 Sungkyunkwan University (SUKKU), 440-746 Suwon, Korea. \\ ${ }^{6}$ Max-Planck-Institut für Radioastronomie, Auf dem Hügel 69, D-53121 Bonn, Germany. \\ ${ }^{7}$ Space Research Institute of RAS, Profsoyuznaya, 84/32, Moscow 117997, Russia.
}

\begin{abstract}
We describe the evolution of of Swift J1644+57, whose unique X-ray properties have led several groups to interpret its behavior as corresponding to an extraordinary event of tidal disruption of a star by a supermassive black hole in the nucleus of a $(z=0.3545)$ galaxy, as derived by GTC. Multiwavelength observations (X-rays, optical, millimetre, centimitre) are proving to be essential to reveal the long term nature of the emission in this source. In particular, we identify for the first time the properties of a forming relativistic jet. In our interpretation of the phenomenon, we leave the still open possibility that it may correspond to the onset of a dormant AGN, but this may only be tested with longer term X-ray, millimetre and centimetre monitoring.
\end{abstract}

\section{Introduction}

On 2011 March 28 Swift triggered on a newly discovered transient, which was initially thought to be a new gamma-ray burst (dubbed GRB 110328A; Cummings et al. 2011). However, a second Swift trigger on this source (Barthelmy et al. 2011) started to cast doubt on the GRB nature of this source, suggesting that instead it may correspond to a new class of hard X-ray transient. This was confirmed by the pre-outburst optical detection in Palomar (Cenko et al. 2011), which strongly disfavored a cosmological long-duration GRB. Furthermore, spectroscopic observations (Levan et al. 2011) revealed the extragalactic nature of the source.

Subsequent radio observations with the EVLA (Zauderer et al. 2011), CARMA (Zauderer et al. 2011), MRAO (Pooley et al. 2011), and PdBI (Castro-Tirado et al. 2011) confirmed the existence of a radio source, coincident with the optical position of the nucleus of a host galaxy, with flux densities at $\mathrm{cm}$ and mm wavelengths of 5-25 mJy, with indication of long term evolution.

All this observational evidence pointed towards the possibility that the source is a very peculiar new type of X-ray transient (Levan et al. 2011), which may be interpreted (Bloom et al. 2011) by a tidal disruption of a main sequence star by a black hole of $10^{6-7}$ solar masses at the center of the observed host galaxy, leading to the formation of a "mini-blazar" with the jet seen face-on. This was also supported by energetic and variability arguments, which suggested that the jet was at least mildly relativistic, presented high collimation, and a spectrum dominated by synchrotron and inverse Compton emission (Bloom et al. 2011).

\section{Multiwavelength observations}

\subsection{X-ray observations}

Target-of-opportunity XMM-Newton observations of the region around $\mathrm{Sw} 1644+57$ were triggered in revolution 2071 (Observation ID. 065840/07). XMM-Newton observed Sw 1644+57 on March 31, 2011 for $27.1 \mathrm{ks}, 3$ days after the onset of the event. Given the X-ray brightness of the source, EPIC-pn was used in Burst mode, whereas the EPIC-MOS1 and MOS2 were used in Small Window and Time mode, respectively. The X-ray lightcurve is shown in Fig. 1.

\subsection{Optical and near-IR observations}

A late epoch 10.4-m Gran Telescopio Canarias optical spectrum (Fig. 2) showed emission lines of $\mathrm{H}_{\alpha}, \mathrm{H}_{\beta}$, [O III], [S II] from which a redshift $z=0.3545 \pm 0.0007$ was derived, confirming the value proposed in Levan et al. (2011), also based on earlier epoch GTC data. See Fig. 1 , None of the emission lines displayed a broad component, as it would be expected for active Seyfert galaxies. 


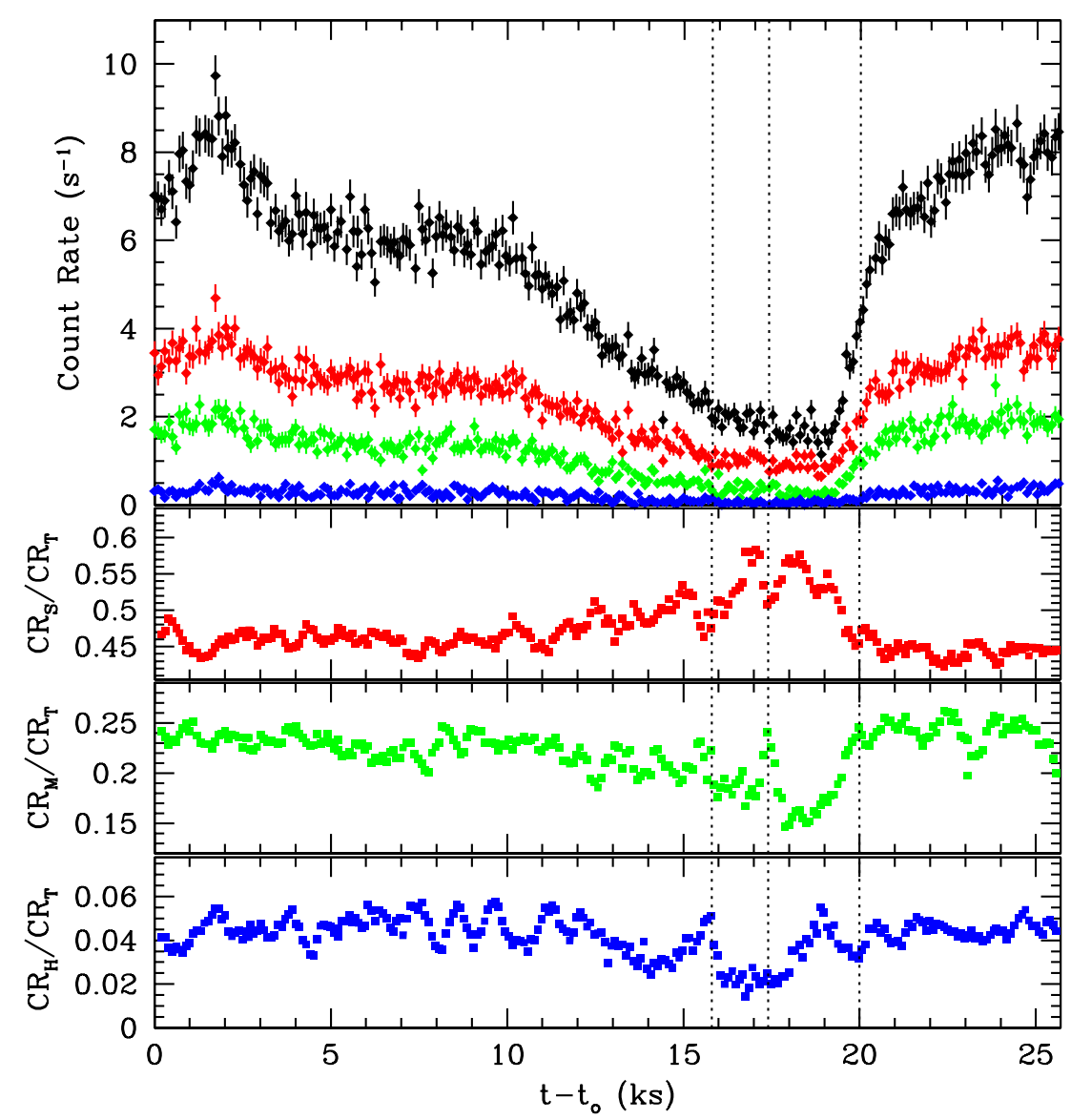

Figure 1. The X-ray lightcurve observed by XMM-Newton as result of the first DDT granted for this particular source (PI : CastroTirado). The relative contributions of the soft (1-2 keV), medium (3-5 keV), and hard (6-10 keV) bands indicate that the spectrum becomes softer when the total flux decreases, also observed in QSOs.

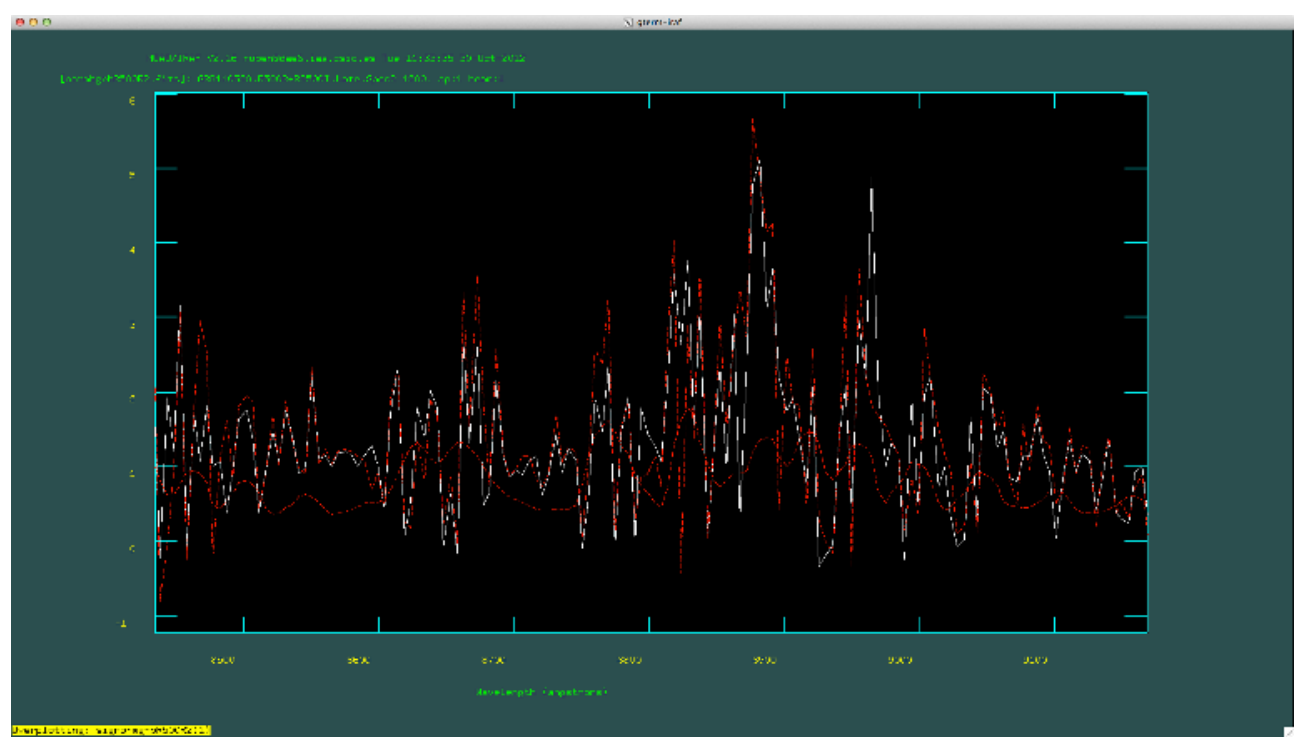

Figure 2. A fraction of the optical spectrum that was obtained on 26 June with the $10.4 \mathrm{~m}$ GTC using the R500R grism (3x900s exposures) in the OSIRIS imaging spectrograph. The R500R grism was used with a spectral range of 5300-10000 A and a dispersion of FWHM $=2.4 \mathrm{~A} /$ pix and a resolution of 634 (both at $7319 \mathrm{~A}$ ). The 1" wide slit was positioned on the location of the transient source and a $2 \times 2$ binning mode was used. 


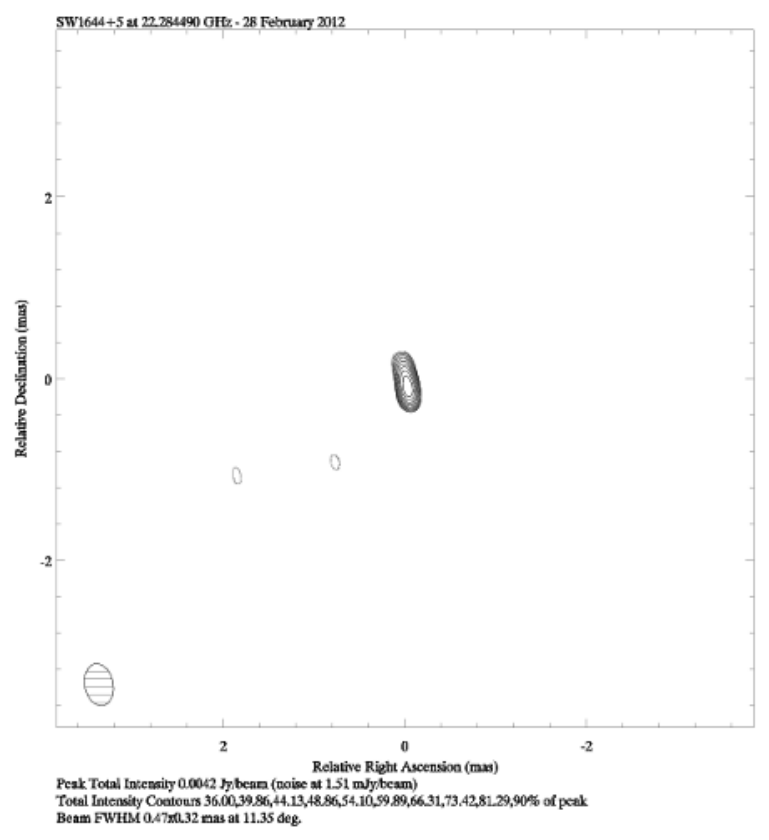

Figure 3. EVN map centred on the Swift J1644+57 showing a clear detection ( $3 \mathrm{mJy}$ at $22 \mathrm{GHz})$ at the target position.

No variations in the line fluxes of the lines were noticeable when comparing to previous measurements, which supports that the line emission is formed in outer star-forming regions existing in the Galaxy. In addition to them, near-IR observations were conducted on Apr 15 and May 21 at the $3.5 \mathrm{~m}$ telescope (+ OMEGA 2000) at the German-Spanish Calar Alto (CAHA) Observatory. Further near-IR observations (in the Ks-band) were conducted on the Apr 15 service night at the $4.2 \mathrm{~m}$ William Herschel Telescope (+ LIRIS) at Observatorio del Roque de los Muchachos, unfortunately poor weather conditions prevented the polarimetric measurements although Sw 1644+57 was detected.

\subsection{Millimetre observations}

Our observations with the Plateau de Bure Interferometer (PdBI) started three days after the onset of the event. The counterpart was detected with an extremely high (>100) $\mathrm{S} / \mathrm{N}$ ratio, on the phase center coordinates (J2000, R.A. = RA : 16 :44 :49.97; Dec : +57:34 :59.7). The observed dual polarisation continuum bandwidth was $1 \mathrm{GHz}$. They showed that, after an initial decay, the flux at $\mathrm{mm}$ wavelengths displayed a significant increase by a factor of more than a factor of 2 during May 2011. PdBI observations are continuing since then, on a monthly basis.

\subsection{Centimetre observations}

Effelsberg observations were attempted on 2011 June 12 (no detection due to poor weather conditions) and on 2011 June 19 , with a marginal detection of $\sim 8 \mathrm{mJy}$ at 4.8 GHz. Additional observations were carried with Korean facilites, also imposing upper limits.

On 2012 February 28 we performed phase-reference observations of Sw1644+57 using the European VLBI
Network (EVN) at a observing wavelength of $1.3 \mathrm{~cm}$. These observations were aimed at detecting any possible proper motion, as well as to resolve its radio structure. By the time of our EVN observations -337 days after the initial burst - the source was still detected at a flux density of $\sim 3 \mathrm{mJy}$ (see Fig. 3).

\section{Discussion}

These (and further) observations led us to suggest that the peculiar X-ray transient Sw 1644+57 may correspond to the onset from a dormant AGN (Castro-Tirado et al. 2013). See Fig. 4. In the case of the AGN onset, we expect that the jet originated by the flaring event that triggered the Swift satellite should conserve its $\mathrm{mm}$ and radio emission on relatively long time scales (of several months more).

In conjunction with other multi-wavelength observations (like the millimetre ones at $\mathrm{PdBI}$ which are being performed routinelly), ongoing (and near future) datasets will allow :

- to search for emission line variability in the optical spectrum, as might have been seen in other events.

- to determine whether the nature of Swift J1644+57 is related to a short time range tidal disruption flare of a star by a supermassive black hole, or to a longer term phenomenon as the onset of a dormant AGN as proposed in Castro-Tirado et al. (2013).

- to perform a detailed study of the long term evolution of the multi-wavelength afterglow emission over the first $\sim 3$ years after the event. This will enable us to trace the evolution of the characteristic synchrotron selfabsorption frequency, and the radio-mm spectral index, that will help help us to constrain among different jet 


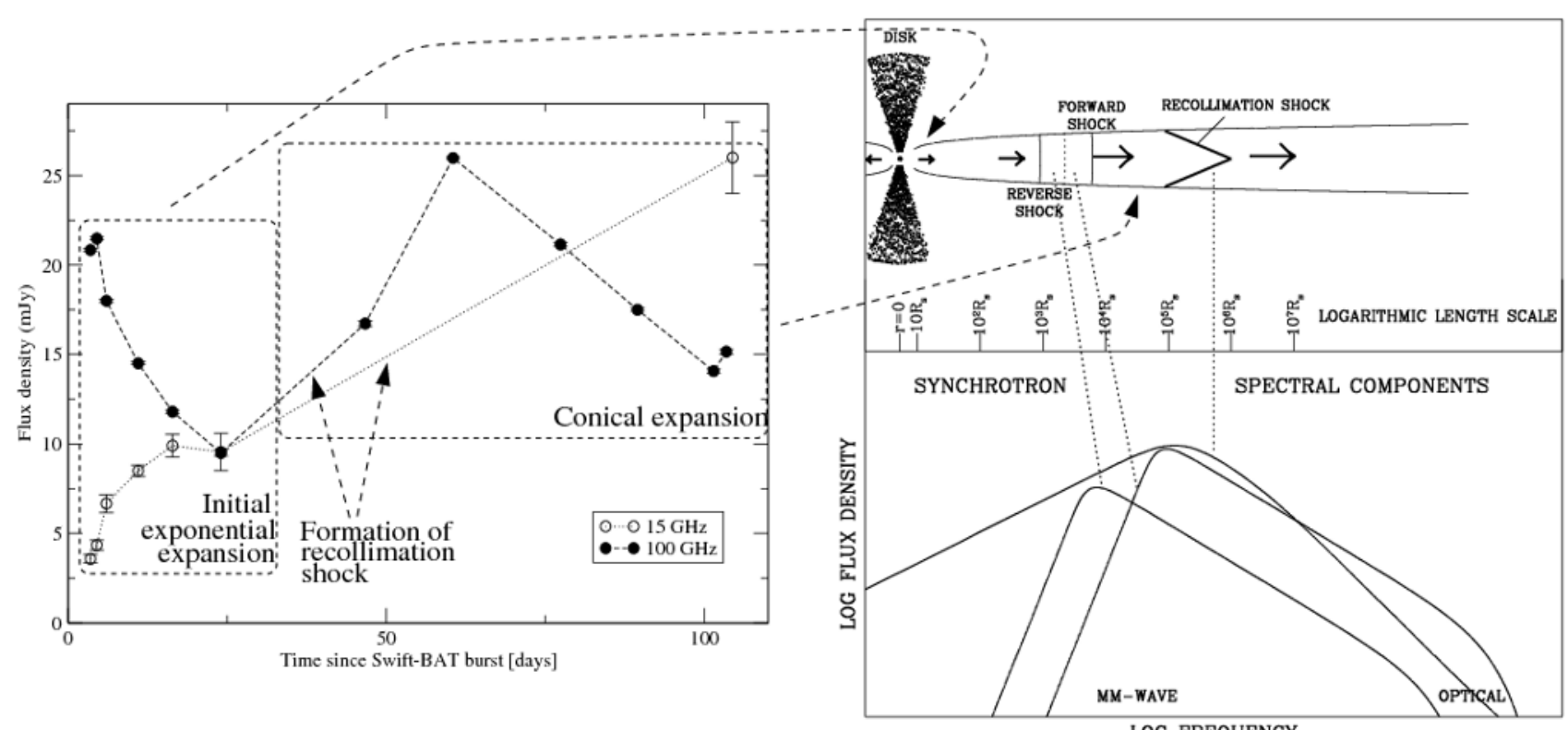

LOG FREQUENCY

Figure 4. The proposed scenario to explain the light curves of Sw 1644+57, following Castro-Tirado et al. 2013. Left : Millimeter and radio light curves of Swift 1644+57. After the initial decay, the mm lightcurve displays a second bursting episode, reaching a maximum flux density even higher than the one observed after the initial Swift detection. Right : Sketch of the main features of the proposed scenario to explain the light curves of Sw 1644+57. The length scale is given in terms of Schwarzschild radii. After the forward shock passes the location where the external pressure becomes nearly constant (rather than increasing toward the black hole), a conical recollimation shock forms and collimates the jet further. Electrons are accelerated to highly relativistic energies at all three shock fronts, creating the three spectral components sketched on the bottom.

models and underlying emission mechanisms (Kumar et al. 2013, Zauderer et al. 2013, Zou et al. 2013).

\section{Acknowledgement}

This work is partly based on observations carried out with the $10.4 \mathrm{~m}$ GTC at the ORM and with the IRAM Plateau de Bure Interferometer. IRAM is supported by INSU/CNRS (France), MPG (Germany) and IGN(Spain).We also acknowledge the support of the Spanish MICINN Project AYA2012-32727-C03-01.

\section{References}

[1] Barthelmy, S. et al. 2011, GCN 11824
[2] Bloom, J. S. et al. 2011, Science, 333, 203

[3] Castro-Tirado, A. J. et al. 2011, GCN 11880

[4] Castro-Tirado, A. J. et al. 2013, Rev. Mex. Astron. Astrof. (Conf. Ser) 42, 36

[5] Cenko, B. et al. 2011, GCN 11827

[6] Cummings et al. 2011, GCN 11823

[7] Kumar, P. et al. 2013, MNRAS 434, 3078

[8] Levan, A. J. et al. 2011, Science, 333, 199

[9] Pooley et al. 2011, GCN 11849

[10] Zauderer, B. A. et al. 2011, Nature 476, 425

[11] Zauderer, B. A. et al. 2013, ApJ 767, 152

[12] Zou, Y. C. et al. 2013, MNRAS 434, 3463 